\title{
Post-transcriptional regulation of glutamate metabolism of Pichia pastoris and development of a glutamate-inducible yeast expression system
}

Trishna Dey and Pundi N Rangarajan*

Department of Biochemistry, Indian Institute of Science, Bangalore 560012, INDIA

*Correspondence to: P. N. Rangarajan, Department of Biochemistry, Indian Institute of Science, Bangalore 560012, INDIA, email: pnr@iisc.ac.in

Pichia pastoris harbours a unique glutamate utilization pathway in which glutamate $\begin{array}{lllllll}\text { dehydrogenase } & 2 & \text { (GDH2), } & \text { aspartate } & \text { aminotransferase } & 2 & \text { (AAT2) and }\end{array}$ phosphoenolpyruvate carboxykinase (PEPCK) catalyze the conversion of glutamate to $\alpha$ ketoglutarate, oxaloacetate, and phosphoenolpyruvate respectively in the cytosol. GDH2 and PEPCK are glutamate-inducible enzymes and their synthesis is regulated posttranscriptionally by Rtg1p, a cytosolic basic helix-loop-helix protein via Rtg1p response elements located downstream of TATA box of GDH2 and PEPCK promoters. Glutamateinducible synthesis of PEPCK is abrogated in $\triangle g d h 2$ and $\Delta a a t 2 . \alpha$-ketoglutarate induces PEPCK synthesis in $\Delta g d h 2$ but not $\Delta a a t 2$. We propose that oxaloacetate derived from glutamate is the inducer of PEPCK synthesis. Enzymes of glutamate utilization pathway are synthesized during carbon starvation and they enable $P$. pastoris to overcome nutritional stress. Finally, green fluorescent protein can be synthesized efficiently from $G D H 2$ and $P E P C K$ promoters using food-grade monosodium glutamate as inducer indicating that the post-transcriptional regulatory circuit described here can be exploited for the development of glutamate-inducible $P$. pastoris expression system. 


\section{Introduction}

Amino acids, in addition to their role as building blocks of proteins, play an important role in several metabolic reactions. In biosynthetic reactions, they directly participate as substrates or undergo deamination and contribute as ammonium ion and keto acids. Glutamate and glutamine serve as nitrogen donors for several nitrogenous compounds in the cell (Magasanik, 1993). In Saccharomyces cerevisiae, NADPH-dependent glutamate dehydrogenases (GDH1, GDH3) convert ammonia and $\alpha$-ketoglutarate into glutamate. The latter combines with ammonia to generate glutamine in a reaction catalyzed by glutamine synthetase. A third, NAD-dependent GDH (GDH2) localized in the mitochondria degrades glutamate to $\alpha$-ketoglutarate and ammonia and thus contributes to anaplerosis of Krebs cycle. Glutamate and glutamine contribute for $85 \%$ and $15 \%$ of total cellular nitrogen, respectively in S. cerevisiae (Miller and Magasanik, 1990) (Mara et al., 2018) (https://www.yeastgenome.org/locus/S000002374\#protein) (Silao et al., 2020). In addition to being a nitrogen donor, Glutamate is also utilized as the sole source of carbon by Pichia pastoris (a.k.a. Komagataella phafii) and several other yeast species (Sahu and Rangarajan, 2016) (Freese et al., 2011). In Scheffersomyces stipitis and P. pastoris, GDH2 is required for the catabolism of glutamate and deletion of $G D H 2$ renders them defective in glutamate utilization (Sahu and Rangarajan, 2016) (Dey et al., 2018) (Freese et al., 2011). In addition to GDH2, aspartate amino transferases (AAT) encoded by AAT1 and AAT2, malate dehydrogenases (MDH) encoded by $M D H 1$ and $M D H 2$ as well as the gluconeogenic enzyme, phosphoenolpyruvate carboxykinase (PEPCK) are also essential for glutamate utilization in $P$. pastoris (Sahu and Rangarajan, 2016) (Dey et al., 2018). Mxr1p, a zinc finger transcription factor and Rtg1p, a basic helix-loop-helix leucine zipper protein regulate the expression of key genes of glutamate utilization pathway of P. pastoris (Sahu and Rangarajan, 2016) (Dey et al., 2018). In S. cerevisiae, Rtg1p heterodimerizes with another basic helix-loop-helix leucine 
zipper protein known as Rtg3p and regulates the expression of specific nuclear genes in glutamate deficient cells with dysfunctional mitochondria, a phenomenon known as retrograde response (Jazwinski, 2014) (Butow and Avadhani, 2004). In P. pastoris, Rtg3p is absent and Rtg1p localizes to cytosol and functions as a post-transcriptional regulator of GDH2 and PEPCK, key enzymes of glutamate utilization pathway (Dey et al., 2018).

\section{Results and discussion}

\section{Identification of Rtg1p response elements in $G D H 2$ and $P E P C K$ promoters}

We had identified several enzymes involved in the utilization of glutamate as the sole source of carbon in P. pastoris (Sahu and Rangarajan, 2016). Of these, GDH2 and PEPCK are glutamate-inducible enzymes and their synthesis from pre-existing mRNAs is regulated by the cytosolic, basic, helix-loop-helix leucine zipper protein, Rtg1p. (Dey et al., 2018). To further understand Rtg1p-mediated regulation of glutamate utilisation pathway of $P$. pastoris, genes encoding epitope tagged GDH2 and PEPCK were expressed from $1.0 \mathrm{~kb}$ of $G D H 2\left(P_{G D H 2}\right)$ and PEPCK $\left(P_{P E P C K}\right)$ promoters respectively (Supplementary Fig. 1) in GS115 as well as mutant strains of $P$. pastoris (Supplementary Table 1). Cells were cultured in a medium containing yeast nitrogen base (YNB) and glucose (YNBD), glycerol (YNBG), acetate (YNBA), ethanol (YNBE) or glutamate (YNB Glu). GDH2 and PEPCK levels in cell lysates were examined by western blotting using anti-epitope tag antibodies while mRNA levels were quantified by qPCR. GDH2 was present only in cells cultured in YNB Glu while PEPCK was detected in cells cultured in YNBG, YNBE, YNBA and YNB Glu with highest levels in cells cultured in YNB Glu (Fig. 1A). Surprisingly, GDH2 mRNA levels were comparable in cells utilizing glucose, glycerol, acetate, ethanol, and glutamate (Fig. 1B). PEPCK mRNA was undetectable during glucose metabolism and it was present in cells utilizing glycerol, ethanol, acetate, and glutamate (Fig. 1B). PEPCK mRNA levels were higher in cells utilizing glutamate and ethanol 
than other carbon sources (Fig. 1B). When $P_{G D H 2}$ and $P_{P E P C K}$ were replaced by the promoter of the gene encoding glyceraldehyde-3-phosphate dehydrogenase $\left(P_{G A P D H}\right)$ (Supplementary Fig. 1), carbon source-specific synthesis of GDH2 and PEPCK was abrogated and they were expressed constitutively in cells cultured in different media (Fig. 1C). Thus, the absence of GDH2 and PEPCK when expressed from their own promoters under certain culture conditions is not due to their instability or degradation. To further understand carbon source-specific regulation of $G D H 2$ and $P E P C K, G F P$ encoding green fluorescent protein was expressed from $P_{G D H 2}$ and $P_{P E P C K}$, GFP was visualized by live cell imaging while mRNA levels were examined by qPCR. GFP was expressed in a carbon source-specific manner (Fig. 1D) and the expression pattern was very similar to that of GDH2 and PEPCK. GFP mRNA levels were similar to GDH2 and PEPCK mRNA levels (compare Fig. 1E with 1B). Analysis of GFP expression from $P_{G D H 2}$ and $P_{P E P C K}$ in $G S 115$ and $\triangle r t g 1$ indicated that GFP protein levels were significantly downregulated in $\triangle r t g 1$ (Fig. 1G) without a corresponding decrease in their mRNA levels (Fig. $1 \mathrm{H})$ indicating that Rtg1p regulates GFP synthesis from $P_{G D H 2}$ and $P_{P E P C K}$ posttranscriptionally.

The results thus far indicate that $\operatorname{Rtg} 1 \mathrm{p}$ is a post-transcriptional regulator of $G D H 2$ and PEPCK suggesting that upstream promoter sequences of $P_{G D H 2}$ and $P_{P E P C K}$ which act as binding sites for transcription factors are unlikely to have a role in Rtg1p-mediated synthesis of GDH2 and PEPCK. We, therefore, focused our attention on promoter sequences downstream of TATA box upto the initiation codon (ATG). In yeast promoters, transcription start site (TSS) is located 40-120 bp downstream of TATA box and it corresponds to the first base of mRNA (McMillan et al., 2019). The region between the TSS and the ATG in the promoter corresponds to the 5' untranslated region (5' UTR) of mRNA which often contains cis-acting elements referred to as riboswitches that bind to small molecules and regulate translation of downstream open reading frames (Garst et al., 2011). Since TSSs of P. pastoris 
GDH2, PEPCK and GAPDH are not characterized, we examined the role of downstream promoter region (DPR) located between the putative TATA box and the initiation codon of $P_{G D H 2}$ and $P_{P E P C K}$ in the Rtg1p-dependent, post-transcriptional regulation (Fig. 2a). DPR of $P_{G A P D H}$ (-99 to $-1 \mathrm{bp}$ ) was replaced with that of $P_{G D H 2}(-86$ to $-1 \mathrm{bp})$ or $P_{P E P C K}(-58$ to $-1 \mathrm{bp})$ (Fig. 2A) and synthesis of GDH2 and PEPCK from these chimeric promoters was examined in lysates of GS115 and $\Delta r t g 1$ cultured in YNB Glu medium by western blotting with anti-epitope tag antibodies. As expected, GDH2 and PEPCK levels were comparable in GS115 and $\Delta r t g 1$ when $P_{G A P D H}$ was used (Fig. $2 \mathrm{~B}, \mathrm{C}$ ). However, when DPR of $P_{G A P D H}$ was replaced with that of $P_{G D H 2}$ or $P_{P E P C K}, \mathrm{GDH} 2$ and PEPCK synthesis became Rtg1p-dependent resulting in the down regulation of protein but not mRNA levels in $\Delta r \operatorname{rg} 1$ (Fig. 2D, E). Thus, the DPRs harbour the cis-acting elements required for Rtg1p-mediated synthesis of GDH2 and PEPCK.

\section{Conversion of glutamate to oxaloacetate is essential for glutamate-inducible synthesis of}

\section{PEPCK}

GDH2 and PEPCK are abundant proteins and can be readily visualized in Coomassie Brilliant Blue-stained SDS polyacrylamide gels of lysates of GS115 cultured in YNB Glu (Fig 3A) (Dey et al., 2018). These protein bands were identified as GDH2 and PEPCK by Mass spectrometry in an earlier study (Dey et al., 2018). As expected, GDH2 and PEPCK were down regulated in $\Delta r t g 1$ cultured in YNB Glu. (Fig. 3A). However, GDH2 was down regulated in $\Delta p e p c k$ and PEPCK was downregulated in $\Delta g d h 2$ (Fig. 3A). These results were confirmed by examining the levels of epitope tagged GDH2 and PEPCK by western blotting of cell lysates using antiepitope tag antibodies (Fig. 3B). GDH2 and PEPCK mRNAs are not downregulated (Fig. 3C) and downregulation of PEPCK in $\Delta g d h 2$ during glutamate metabolism was observed only during the utilization of glutamate but not acetate and ethanol (Fig. 3D). The first three reactions of glutamate catabolism catalyzed by GDH2, AAT2 and PEPCK result in the 
generation of $\alpha$-ketoglutarate (Akg), oxaloacetate (Oaa) and phosphoenolpyruvate in the cytosol (Fig. 3E) (Sahu and Rangarajan, 2016). We hypothesized that glutamate may not be the direct inducer of PEPCK synthesis. To test this hypothesis, we examined the ability of Akg to induce GDH2 and PEPCK synthesis. Akg readily induced the synthesis of PEPCK but not GDH2 (Fig. 3F). Akg also induced GFP expression from $P_{P E P C K}$ but not $P_{G D H 2}$ (Fig. 3G) suggesting that inducers of GDH2 and PEPCK synthesis are different. Since the synthesis of Akg is impaired in $\Delta g d h 2$, we examined Akg-inducible PEPCK synthesis in $\Delta g d h 2$. GS115 and $\Delta g d h 2$ expressing Myc-tagged PEPCK were cultured in YNB Glu or YNB Akg and PEPCK protein levels were examined by western blotting. Only Akg but not glutamate induced PEPCK synthesis in $\Delta g d h 2$ (Fig. 3H). Since Akg is converted to Oaa by AAT2 and Oaa is the substrate of PEPCK, we examined the ability of Oaa to induce PEPCK synthesis. However, PEPCK synthesis was not induced in cells cultured in YNB Oaa (data not shown). We therefore examined the ability of Oaa synthesized intracellularly via AAT2-catalyzed reaction to induce PEPCK synthesis. Both glutamate- and Akg-inducible synthesis of PEPCK was abrogated in $\triangle a a t 2$ (Fig. 3I) suggesting that Oaa is the inducer of PEPCK synthesis. Based on these results, we propose a model for the post-transcriptional regulation of $P$. pastoris glutamate utilization pathway wherein $G D H 2^{D P R}$ and $P E P C K^{D P R}$ contain cis-acting elements named Rtg1p response elements (RRE) between the transcription start site and initiation codon. Transcription results in the generation of GDH2 and PEPCK mRNAs harbouring the RREs in their 5' UTRs (5' $\mathrm{UTR}^{\mathrm{RRE}}$ ). Translation of $G D H 2$ mRNA is facilitated by the interaction of glutamate and Rtg1p with the 5' $\mathrm{UTR}^{\mathrm{RRE}}$ of $G D H 2$ mRNA while PEPCK synthesis requires interactions amongst Rtg1p and oxaloacetate and 5' UTR ${ }^{\mathrm{RRE}}$ of PEPCK mRNA (Fig. 3J).

\section{Glutamate catabolism confers tolerance to carbon starvation}

The results presented thus far demonstrate that amino- or keto-acids added to the culture medium can induce the translation of GDH2 and PEPCK mRNAs in P. pastoris. GDH2 is 
known to funnel the carbon skeletons of glutamate into the TCA cycle for energy production under carbon limiting conditions (Miyashita and Good, 2008). Yeasts such as P. pastoris are likely to experience nutrient starvation in their natural habitats and they evolve several strategies to survive under these conditions. We reasoned that intracellular metabolites such as amino- and keto-acids generated during carbon starvation may induce the synthesis of GDH2 and PEPCK to facilitate energy generation and gluconeogenesis. P. pastoris cells expressing epitope-tagged GDH2 and PEPCK were cultured in nutrient-rich medium and then shifted to starvation medium which is completely devoid of an exogenous carbon source. Synthesis of GDH2 and PEPCK proteins from pre-existing mRNAs was induced within $6 \mathrm{~h}$ of carbon starvation (Fig. 4A, B). Carbon starvation results in the activation of autophagy which in turn results in the generation metabolites needed for energy generation, biosynthetic reactions, homeostasis and survival (Iwama and Ohsumi1, 2019) (Adachi et al., 2017) (Weber et al., 2020). To examine whether the translation of $G D H 2$ and PEPCK mRNAs is triggered by the metabolites generated upon induction of autophagy during carbon starvation, we treated cells with methionine, a known inhibitor of non-nitrogen starvation induced autophagy (Sutter et al., 2013) and analyzed GDH2 and PEPCK protein levels by western blotting. Synthesis of PEPCK but not GDH2 was impaired by methionine treatment (Fig. 4C). Thus, inducers of GDH2 and PEPCK synthesis are generated by autophagy-independent and -dependent pathways during carbon starvation, respectively. We next compared the viability of GS115, $\Delta g d h 2$ and $\Delta p e p c k$ during carbon starvation by cell survival assay. GS115 expressing GDH2 and PEPCK exhibited greater viability than $\Delta g d h 2$ and $\Delta p e p c k$ when observed over a period of two weeks of carbon starvation (Fig. 4D, E). The decrease in viability was much more pronounced in case of $\triangle p e p c k$ than $\Delta g d h 2$. Thus, starvation-induced synthesis of GDH2 and PEPCK from pre-existing mRNAs enables $P$. pastoris to rapidly respond to extreme carbon starvation conditions and overcome nutritional stress. 


\section{A glutamate-inducible $P$. pastoris expression system}

The promoter of AOXI $\left(P_{A O X I}\right)$ encoding alcohol oxidase I is widely used for methanolinducible production of heterologous proteins in P. pastoris (Hartner and Glieder, 2006) (Cereghino and Cregg, 2000). Methanol is toxic and flammable compound, needs special handling and is not recommended to produce certain edible and medical products. Monitoring methanol concentration in $P$. pastoris cultures is necessary to prevent accumulation of formaldehyde and $\mathrm{H}_{2} \mathrm{O}_{2}$, by-products of methanol metabolism, to toxic levels necessitating the development of methanol-free $P_{A O X I}$-based expression systems (Vogl et al., 2018) (Wang et al., 2017) (Shen et al., 2016) (Prielhofer et al., 2013). Thus, there is a need to develop expression systems which avoid methanol but maintain the high productivity of $P$. pastoris. Food grade monosodium glutamate (MSG) is more readily soluble in water than glutamate, generally regarded as safe (GRAS) by USFDA, available in large quantities, and used as a flavour enhancer in several Asian cuisines (https://www.amazon.in/Ajinomoto-MonosodiumGlutamate-Umami-Seasoning/dp/B00IH28XDE) (https://www.fda.gov/food/food-additivespetitions/questions-and-answers-monosodium-glutamate-msg). MSG readily induced GFP expression from $P_{G D H 2}$ and $P_{P E P C K}$ (Fig. 5A). P. pastoris strain expressing GFP from $P_{A O X I}$ was constructed and GFP synthesis from methanol-inducible $P_{A O X I}$ was compared with that from MSG-inducible $P_{G D H 2}$ and $P_{P E P C K}$. Cells were cultured overnight in shake flasks in YNBD medium and then shifted to YNB and methanol (YNBM) or YNB Glu. After $24 \mathrm{~h}$, cells were lysed and GFP levels were examined by western blotting using anti-GFP antibody (Fig. 5B). In another experiment, whole cell lysates were incubated with GST-tagged anti-GFP nanobodies (Katoh et al., 2015), proteins were pulled down using glutathione resin, and visualized on SDS polyacrylamide gel by Coomassie Brilliant Blue staining (Fig. 5C). Western blot and Coomassie blue-stained SDS gel were scanned and GFP was quantitated. The results indicate that GFP expression from $P_{G D H 2}$ and $P_{P E P C K}$ is $65 \%$ and $90 \%$ respectively of that from 
$\mathrm{P}_{\text {AOXI }}$ (Fig. 5D). GFP was also quantified using a standard curve prepared using known quantities of BSA. GFP expression from $P_{G D H 2}$ and $P_{P E P C K}$ and $\mathrm{P}_{A O X I}$ was $8.3,12.6$ and 14.4 $\mu \mathrm{g}$ per $1.2 \mathrm{ml}$ of culture (Fig. 5E).

The efficiency of the glutamate-inducible expression system described here can be further improved by expressing the gene of interest from both $P_{G D H 2}$ and $P_{P E P C K}$ (Fig. 5F). The combined strength of $P_{G D H 2}$ and $P_{P E P C K}$ is likely to be more than that of $P_{A O X I}$. While GDH2 is expressed only in cells utilizing glutamate, PEPCK is synthesised in cells utilizing glutamate as well as ethanol, acetate and glycerol albeit at varying levels. (Fig. 1A-D). Thus, by choosing an appropriate carbon source, one can express a heterologous gene at low, moderate, or high levels from $P E P C K$ promoter which can be of great importance in expressing proteins that are cytotoxic at high levels as well as expressing proteins of a metabolic pathway at variable levels during metabolic engineering. The high-density fermentation strategy currently being employed for methanol-inducible production of recombinant proteins can be readily adapted for glutamate-inducible expression with only minor modifications. The expression system described here is unique as recombinant protein production involves post-transcriptional regulation. GDH2- and PEPCK promoter-based P. pastoris expression system will be useful for both academic research and industrial applications.

Taken together, this study demonstrates that the glutamate utilization pathway is of great physiological significance. Glutamate-inducible synthesis of GDH2 and PEPCK is regulated by Rtg1p in conjunction with their respective substrates and RREs. The fact that the translation of GDH2 and PEPCK is highly regulated and they express at high levels in cells cultured in YNB Glu medium has been exploited for the development of a novel, glutamateinducible $P$. pastoris expression system for the synthesis of heterologous proteins as an alternative to the methanol-inducible expression system. 


\section{Materials and methods}

\section{Media and culture conditions}

A single colony of yeast cells was inoculated from agar (2.0\%) plates containing YPD (1.0\% yeast extract, $2.0 \%$ peptone, $2.0 \%$ glucose) into YPD liquid medium and grown overnight at $30^{\circ} \mathrm{C}$ in an orbital shaker at $180 \mathrm{rpm}$. Cells were washed with sterile distilled water, atleast twice, and shifted to different minimal media containing $0.17 \%$ yeast nitrogen base (YNB) without amino acids and with $0.5 \%$ ammonium sulphate supplemented with $2.0 \%$ glucose (YNBD), $2.0 \%$ glycerol (YNBG), $1.0 \%$ ethanol (YNBE), $2.0 \%$ acetate (YNBA), $1.0 \%$ methanol (YNBM) or 1.0\% glutamate (YNB Glu).

\section{Yeast and bacterial strains}

Yeast strains used in this study are listed in Supplementary Table1. Yeast cells were transformed by electroporation (Gene Pulser, Bio-Rad, CA). Escherichia coli DH5 $\alpha$ strain was used for cloning of recombinant plasmids and transformation was done by $\mathrm{CaCl}_{2}$ method.

\section{Antibodies and other reagents}

Oligonucleotides were purchased form Sigma-Aldrich (Bangalore, India). Mouse anti-Myc tag antibody was purchased from Merck Millipore (Bangalore, India). Mouse anti-GFP was purchased from Santa Cruz Biotechnology Inc. (Santa Cruz, CA) and mouse anti-His tag antibodies were purchased from cell signalling and technology (Denvers, MA). Antiphosphoglyerate kinase (PGK) antibody was generated by immunizing rabbit with purified $P$. pastoris PGK protein. Restriction enzymes and T4 DNA ligase were purchased from New England Biolabs (Ipswich, MA). DNA polymerases were purchased from GeNei (Bangalore, India) and Thermo Fisher Scientific (Waltham, MA). 


\section{Total RNA isolation, Reverse Transcription-PCR (RT-PCR) and Quantitative PCR (qPCR)}

Total RNA was isolated from P. pastoris by hot-phenol method (Schmitt et al., 1990). Yeast cells grown in a specified medium were harvested by centrifugation ( $3000 \mathrm{x} \mathrm{g}$ for $5 \mathrm{~min}$ ) at room temperature. Cells were resuspended in $400 \mu \mathrm{l}$ of $\mathrm{AE}$ buffer $(50 \mathrm{mM}$ sodium acetate, $\mathrm{pH}$ 5.3 and $10 \mathrm{mM}$ EDTA, pH 8). To this $40 \mu \mathrm{l}$ of $10 \%$ SDS was added and vortexed briefly. After vortexing, $500 \mu \mathrm{l}$ of $\mathrm{AE}$ buffer-saturated phenol was added, vortexed and heated at $65^{\circ} \mathrm{C}$ for 4 min. The tubes were then snap chilled in liquid nitrogen for $1 \mathrm{~min}$ and centrifuged $(10000 \mathrm{x} \mathrm{g}$ for $15 \mathrm{~min}$ ) for the separation of aqueous and organic phases. The aqueous phase was carefully transferred to a fresh tube followed by phenol-chloroform extraction in which equal volume of phenol-chloroform (1:1) was added, vortexed briefly and centrifuged (10000 x g for $5 \mathrm{~min})$ at room temperature. To the upper aqueous phase, $40 \mu \mathrm{l}$ of $3 \mathrm{M}$ sodium acetate ( $\mathrm{pH}$ 5.3) and 2.5 volumes of chilled $100 \%$ ethanol was added, mixed thoroughly by inverting and incubated at $20^{\circ} \mathrm{C}$ for 30 min for the precipitation of RNA. RNA pellet was obtained by centrifugation at $10000 \mathrm{x}$ g for $15 \mathrm{~min}$ at $4^{\circ} \mathrm{C}$. RNA pellet was washed with chilled $80 \%$ ethanol, air dried and resuspended in water. For quantitation, absorbance of RNA was recorded at $260 \mathrm{~nm}$. cDNA was prepared using $1 \mu \mathrm{g}$ of DNase treated RNA followed by q-PCR using StepOnePlus RealTime PCR system (Thermo Fisher Scientific).

\section{Western blotting}

Whole cell protein extracts were made by either glass bead lysis or trichloro- acetic acid (TCA) precipitation method. For TCA precipitation, briefly $5.0 \mathrm{OD}_{600}$ units of $P$. pastoris cells cultured in specified medium were centrifuged (Hettich, $3000 \mathrm{x} \mathrm{g}$ for $5 \mathrm{~min}$ ) at room temperature, resuspended in $1 \mathrm{ml} 20 \%$ TCA, vortexed thoroughly and centrifuged again (3000 $\mathrm{x} g$ for $5 \mathrm{~min}$ ) at room temperature. TCA was removed completely and to the pellet $200 \mu \mathrm{l} 20 \%$ 
TCA was added. Acid washed $0.5 \mathrm{~mm}$ glass beads (Biospec Products) were added in 1:1 ratio (w/w) and vortexed (at highest setting) 4 times with intermittent chilling on ice. The slurry was aspirated in fresh microfuge tube and TCA was removed by centrifugation $\left(10000 \mathrm{x} \mathrm{g}\right.$ at $4^{\circ} \mathrm{C}$ for $15 \mathrm{~min}$ ). Brief spin was given again to remove left over TCA. 1X Laemmli buffer (50 mM Tris-HCl, $\mathrm{pH}$ 6.8, 2\% SDS, $0.1 \%$ bromophenol blue, $10 \%$ glycerol and $100 \mathrm{mM} \beta$ mercarptoethanol) was added to the pellet, vortexed, heated for $5 \mathrm{~min}$ at $95^{\circ} \mathrm{C}$ and vortexed again. Final whole cell protein lysate was extracted from left over debris by centrifugation (10000 x g at $4^{\circ} \mathrm{C}$ for $\left.10 \mathrm{~min}\right)$. Lysate volume corresponding to $0.5 \mathrm{OD}_{600}$ units of cells was resolved on SDS-PAGE. For Glass bead mediated lysis, yeast cells were resuspended in lysis buffer $(1: 1 \mathrm{w} / \mathrm{v})$ containing $20 \mathrm{mM}$ Tris $(\mathrm{pH} 8.0), 400 \mathrm{mM} \mathrm{NaCl}, 10 \mathrm{mM} \mathrm{MgCl} 2,10 \mathrm{mM}$ EDTA (pH 8.0), $10 \%$ glycerol, $7 \mathrm{mM} \beta$-mercaptoethanol and protease inhibitor cocktail (Roche complete, EDTAfree Protease Inhibitory Cocktail tablets, Manheim, Germany). Acid washed $0.5 \mathrm{~mm}$ glass beads (Biospec Products) were added in 1:1 ratio (w/w) and vortexed 10 times using a vortex mixer (at highest setting) with intermittent chilling on ice. Cell debris were removed by centrifugation ( $10000 \mathrm{x}$ g at $\left.4^{\circ} \mathrm{C}\right)$. Proteins were estimated using Bradford reagent (BioRad) and resolved on SDS-PAGE, electroblotted onto a $0.22 \mu \mathrm{m}$ PVDF membrane using transfer buffer (39 mM glycine, $48 \mathrm{mM}$ Tris ( $\mathrm{pH} \mathrm{8.0),} \mathrm{20 \%} \mathrm{methanol).} \mathrm{The} \mathrm{membrane} \mathrm{was}$ blocked in 5\% non-fat milk for $1 \mathrm{~h}$, prepared in TTBS (25 mM Tris (pH 8.0), 0.1\% Tween 20 and $125 \mathrm{mM} \mathrm{NaCl}$ ) followed by three washes in TTBS for $5 \mathrm{~min}$. Blots were sequentially incubated in TTBS containing antibodies of appropriate dilution, raised against a specific protein or anti-epitope tag antibodies, for $1 \mathrm{~h}$ either at room temperature or overnight at $4^{\circ} \mathrm{C}$ and washed in TTBS (thrice for $5 \mathrm{~min}$ ). Primary antibodies were detected by HRP-conjugated antirabbit/anti-mouse $\operatorname{IgG}\left(1: 10000\right.$ dilution, $1 \mathrm{~h}$ and $4^{\circ} \mathrm{C}$ incubation). Proteins were detected using Immobilon Western Chemiluminescent HRP substrate (Millipore MA) as per the manufacturer's instructions. 


\section{Live cell imaging}

For live cell imaging, $3 \mu \mathrm{l}$ of growing culture was layered on top of an agarose pad and allowed to settle for 1 min. Finally, on top of the cell suspension coverslip was placed and sealed with transparent nail paint. GFP expression in cells was then visualised using confocal microscope (Olympus FLUOVIEW FV3000). To make agarose pad, glass slides were wiped thoroughly and both ends were fixed with scotch tape. $30 \mu \mathrm{l}$ of molten $1.0 \%$ agarose (in 1X PBS) was placed on top of the glass slide into a round droplet. Another clean slide was placed on top of the agarose droplet and pressed gently to make the flattened agarose pad. This entire set up of the agarose pad was lifted carefully and placed on top of a precooled metal block for $1 \mathrm{~min}$. After the solidification of agarose, the glass slides were separated by gentle sliding motion leaving the agarose pad stuck to one of the two slides which is used further.

\section{Cell viability assay}

From the frozen stock, strains were streaked onto YPD plates and incubated at $30^{\circ} \mathrm{C}$ for $2-3$ days. Single colony was picked and inoculated in YPD medium. Cells were grown in YPD at $180 \mathrm{rpm}, 30^{\circ} \mathrm{C}$ till late stationary phase (2 days). YPD grown late stationary phase cells were transferred to starvation medium i.e., $0.17 \%$ YNB without amino acids and with $0.5 \%$ ammonium sulphate supplemented with $20 \mu \mathrm{g} / \mathrm{ml}$ of histidine with initial OD $_{600}$ of $\sim 0.5$ per ml of media. At specified time points, $\mathrm{OD}_{600}$ was recorded and adjusted to $\sim 0.1$ per ml. 1:10 serial dilutions were made and $50 \mu 1$ of $10^{-3}$ dilution was spread on YPD plates. Plates were incubated at $30^{\circ} \mathrm{C}$ for 2-3 days and colony forming unit per $\mathrm{ml}(\mathrm{CFU} / \mathrm{ml})$ was calculated as a readout for cell survival. CFU per ml at day zero was considered $100 \%$.

Generation of $P$. pastoris $G S 115$ and $\triangle r t g 1$ expressing $G D H 2^{H i s}$ and $P E P C K^{M y c}$ from $P_{G D H 2}$ and $P_{P E P C K}$, respectively, $\triangle g d h 2$ expressing $P E P C K^{M y c}$ from $P_{P E P C K}, \triangle p e p c k$ expressing 


\section{$G D H 2^{H i s}$ from $P_{G D H 2,} \triangle g d h 2$ expressing $P E P C K^{M y c}$ from $P_{P E P C K}$ and $\triangle a a t 2$ expressing $P_{E P C K^{M y c}}$ from $P_{P E P C K}$}

Expression cassette comprising of genes encoding PEPCK along with $1 \mathrm{~kb}$ of its promoter was cloned into pIB3 vector (cat \# 25452, Addgene, USA) and expressed in P. pastoris GS115, $\Delta r t g 1, \Delta g d h 2$ and $\triangle a a t 2$ as Myc-tagged proteins. GDH2 was cloned with 545 bp of its promoter in $p I B 3$ vector as a His-tagged protein and expressed in P. pastoris GS115, Artgl and Apepck. PEPCK was amplified from GS115 genomic DNA using primer pair 5' GGGGTACC CACCCACCCTCAAGTGC 3' and 5' CCCAAGCTTCTACAGGTCTTCTTCAGAGATC AGTTTCTGTTCC AACTGAGGGCCGGCCTG 3' (KpnI and HindIII sites in the primers are underlined, respectively). GDH2 was amplified from GS115 genomic DNA using primer pair 5' CCGGAATTCCTCTCATGTTCGGATAATTCCAGCGGCTTTC 3' and 5' CCGCTCGAG CTAATGATGATGATGATGATGCAATCC CCGAGACTTGTAC 3' (EcoRI and XhoI sites are underlined in the primers, respectively). PCR products were cloned into $p I B 3$ vector and

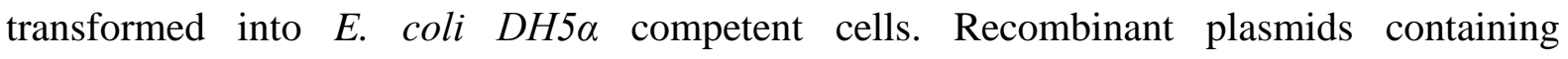
$P_{P E P C K} P E P C K-M y c$ and $P_{G D H 2} G D H 2-H i s$ were linearized using BsrGI and StuI, respectively and transformed into respective $P$. pastoris strains by electroporation. Recombinant clones were selected by plating on YNBD His ${ }^{-}$plates and clones expressing Myc-tagged PEPCK and His-tagged GDH2 were confirmed by western blotting using anti-Myc and anti-His antibodies.

Generation of $P$. pastoris $G S 115$ and $\triangle r t g 1$ strains expressing GFP from $P_{G D H 2}$ and $P_{P E P C K}$ Gene encoding GFP was cloned under $1 \mathrm{~kb}$ promoter of $G D H 2$ and PEPCK into pIB3 vector (Addgene plasmid \#25452) and expressed in P. pastoris GS115 and P. pastoris Artg1 (4rtgl). For the generation of $P_{G D H 2}-G F P$ construct, $1 \mathrm{~kb} G D H 2$ promoter was amplified from $G S 115$ genomic DNA using primer pair 5' CCGCTCGAGGGACAACCAAAGCATCC 3' and 5' CTC CTTTACTAGTCAGATCTACCATAGTGGGTTGGGAGTTTAGTGG 3'. The coding region 
of GFP was amplified from the vector, pREP41GFP (Craven et al., 1998) using primer pair 5' CCACTAAACTCCC AACCCACTATGGTAGATCTGACTAGTAAAGGAG $3^{\prime}$ and $5^{\prime}$ CCCAAGCTtCtagtgG TGGTGGCTAGCTTTG 3'. The amplified GDH2 and GFP products were then purified and used as templates in the final PCR using primer pair 5' CCGCTCGAGGGACAACCAAAGCATCC CCCAAGCTTCTAGTGGTGGTGGCTAGCTTTG 3' (XhoI and HindIII sites are underlined in the primers, respectively). The overlapping product was digested and ligated in pIB3 vector followed by transformation in $E$. coli DH5 $\alpha$ competent cells. For the generation of $P_{P E P C K}-G F P$ construct, $1 \mathrm{~kb}$ PEPCK promoter was amplified from GS115 genomic DNA using primer pair 5' GGGGTACCCACCCACCCTCAAGTGC $\quad 3^{\prime}$ and 5' CCTTCTCATAG ATTATTATCCACAATGGTAGATCTGACTAGTAAAGGAG 3'. GFP was amplified using primer pair 5' CTCCTTTACTAGTCAGATCTACCATTGTGGATAATAATCTATGAGAA GG 3' and 5' CCCAAGCTTCTAGTGGTGGTGGCTAGCTTTG 3'. The amplified PEPCK and GFP products were then purified and used as templates in the final PCR using primer pair 5' GGGGTACCCACCCACCCTCAAGTGC 3' and 5' CCCAAGCTTCTAGTGGTGGTGGC TAGCTTTG 3' (KpnI and HindIII sites are underlined in the primer respectively). The overlapping product was digested and ligated in $p I B 3$ vector followed by transformation in $E$. coli DH5 $\alpha$ competent cells. Both the recombinant plasmids were linearised with SalI and transformed by electroporation into GS115 and $\Delta r \operatorname{tg} 1$. Recombinant clones were selected by plating on YNBD His ${ }^{-}$plates and clones expressing GFP were confirmed by western blotting using anti-GFP antibody.

\section{Generation of GS115 strain expressing GFP from $P_{A O X I}$}

For the construction of $P_{A O X 1}-G F P, 1 \mathrm{~kb}$ of AOX1 promoter was amplified by PCR from $P$. pastoris genomic DNA using the primer pair 5' CGGGGTACCTCATGTTGGTATTGTGAA ATAGACGCAGATC 3' and 5' CTCCTTTACTAGTCAGATCTACCATCGTTTCGATAAT 
TAGTTGTTTTTTGATC 3'. KpnI site is underlined. GFP ORF was amplified from pREP4lGFP (Craven et al., 1998) using the primer pair 5' GATCAAAAAACAACTAATTATTCGAAACGATGG TAGATCTGACTAGTAAAGGAG 3' and 5' CCGCTCGAGCTAGTGGTGGTGGCTAGCT TTG 3'. XhoI site is underlined. In the third and final PCR reaction, the PCR products from the first two reactions were used as templates and amplified using 5' CGGGGTACCTCATGTTGG TATTGTGAAATAGACGCAGATC 3' and 5' CCGCTCGAGCTAGTGGTGGTGGCTAGC TTTG 3' primers to generate $P_{A O X^{-}} G F P$ expression cassette, which was digested and cloned into $p I B 3$ to generate $p I B 3-P_{A O X}-G F P$. The recombinant plasmid was linearized with SalI, transformed into GS115 and plated on YNBD His`agar plates. Colonies expressing GFP were identified by western blotting of cell lysates using anti-GFP antibodies.

Generation of $P$. pastoris $G S 115$ and $\triangle r t g 1$ strains expressing $G D H 2^{H i s}$ and $P E P C K^{M y c}$

\section{from $P_{G A P D H}$}

For the generation of $P_{G A P D H}-G D H 2^{H i s}$ and $P_{G A P D H}-P E P C K^{M y c}$ expression cassettes, genes encoding GDH2 and PEPCK were amplified from GS115 genomic DNA and cloned into pGHYB vector (Addgene plasmid \#87447) (Yang et al., 2014) under GAPDH promoter. For GDH2 amplification 5' CCGCTCGAGATGGTCGACAGACTCCAAGTGTC $3{ }^{\prime}$ and 5' ATAAGAATGCGGCCGC CAATCCCCGAGACTTGTACTC 3' primer pair was used (XhoI and NotI sites are underlined respectively). For PEPCK amplification 5' CCGCTCGAGATGGCTCCTACTGCTATAGATTTAC $\quad 3^{\prime}$ and ATAAGAATGCGGCCGCCTACAGGTCTTCTTCAGAGATCAGTTTCTGTTCCAACTG AGGGCCGGCCTG 3' primer pair was used (XhoI and NotI sites are underlined, respectively). Both the recombinant plasmids were linearised with AvrII and transformed by electroporation

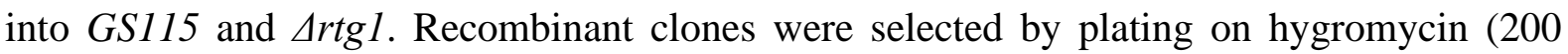


$\mu \mathrm{g} / \mathrm{ml}$ ) containing YPD plates and clones expressing the epitope tagged proteins were confirmed by western blotting using anti-His and anti-Myc antibodies.

Generation of $P$. pastoris $G S 115$ and $\triangle r t g 1$ strains expressing $G D H 2^{H i s}$ and $P E P C K^{M y c}$ from $P_{G A P D H}$ containing $G D H 2^{D P R}$ and $P E P C K^{D P R}$

To generate $P_{G A P D H-G D H 2}{ }^{D P R} G D H 22^{H i s}$ expression cassette, GAPDH promoter (Supplementary Fig. 1) from - 1045 To -106, just upstream its TATAA box and $G D H 2$ from its putative TATAA box (-94) to stop codon were amplified from GS115 genomic DNA. GDH2 was amplifies with 6X-His tag sequence upstream its stop codon. GAPDH promoter was amplified using 5' GGGGTACCGAAGTAAA ACTTTAACTTCAG 3' and 5' CTGGGCGATCTGGAGCTATT TATATTCGATTCTGGTG GTTTCCAATAATC 3' primer pair. GDH2 was amplified using primer pair, 5' GATTATTGGAAACCACCAGAATCGAATATAAATAGCTCCAGATCGC CCAG 3' and 5' CCGCTCGAGCTAATGATGATGATGATGATGCAATCCCCGAGACTT GTAC 3'. The PCR products were used as templates for final PCR using 5' GGGGTACCGAA GTAAAACTTTAACTTCA G 3' and 5' CCGCTCGAGCTAATGATGATGATGATGATGC AATCCCCGAGACTTGTA C 3' primer pair (KpnI and XhoI sites are underlined respectively) and cloned into pIB3 vector (cat \# 25452, Addgene, USA). Similar strategy was used for $P_{\text {GAPDH-PEPCK }}{ }^{D P R} P E P C K^{M y c}$ construct generation. Primer pair 5' GGGGTACCGAAGTAAAA CTTTAACTTCAG 3' and 5' GGCGGCCAGCCTGCCCCTATTTATATTCGATTCTGGTG GTTTCCAATAATC 3' was used for GAPDH promoter (from -1045 to -106, just upstream its TATA box) amplification and primer pair 5' GATTATTGGAAACCACCAGAATCGAAT ATAAATAGGGGCAGGCTGGCCGCC 3' and 5' CCCAAGCTTCTACAGGTCTTCTTCA GAGATCAGTTTCTGTTCCAACTGAGGGCCGGCCTG 3' was used for PEPCK (from -66 bp to stop codon) amplification. Final PCR was done to generate the desired construct using

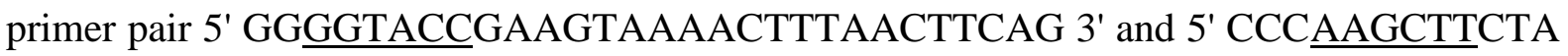
CAGGTCTTCTTCAGAGATCAGTTTCTGTTCCAACTGAGGGCCGGCCTG 3' (KpnI and 
HindIII sites are underlined respectively). Both the recombinant plasmids were linearised with StuI and transformed by electroporation into GS115 and $\Delta r t g 1$. Recombinant clones were selected by plating on YNBD His ${ }^{-}$plates and clones expressing the epitope tagged proteins were confirmed by western blotting using anti-His and anti-Myc antibodies.

\section{GST Pull down assay}

E. coli cells (BL21DE3) expressing GST tagged anti-GFP nanobody (Addgene plasmid \#61838) (Katoh et al., 2015) were suspended in 1X PBS (137 mM NaCl, $2.7 \mathrm{mM} \mathrm{KCl,} 100$

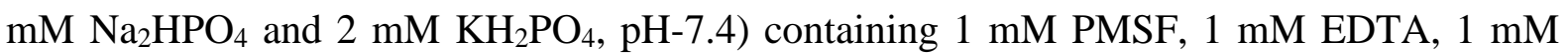
DTT, $10 \mu \mathrm{g} / \mathrm{ml}$ lysozyme and $1 \%$ Triton X-100 and kept on ice for $20 \mathrm{~min}$. Cells were then sonicated and cell lysate containing GST tagged anti-GFP nanobody was incubated with glutathione resin (G-Biosciences, U.S.A.) at $4^{\circ} \mathrm{C}$ for $1 \mathrm{~h}$. Nanobody bound glutathione resins were harvested by brief centrifugation followed by washes with 1X PBS containing $1 \mathrm{mM}$ PMSF. GST tagged anti-GFP nanobody bound glutathione resins were incubated with yeast (GS115) whole cell protein lysate containing GFP expressing from either $P_{A O X 1}, P_{G D H 2}$ or $P_{P E P C K}$ at $4^{\circ} \mathrm{C}$ for $2-3 \mathrm{~h}$. Post interaction resins were centrifuged briefly and washed atleast thrice with cold $1 \mathrm{X}$ PBS. Bound proteins were resolved by SDS-PAGE and visualized by Coomassie Brilliant Blue R staining.

\section{Statistical analysis}

The relevant statistical test and replicate type for each figure are found in the corresponding figure legends.

\section{Data availability}


The authors declare that all data supporting the findings of the present study are available in the article and its supplementary figures and tables, or from the corresponding author upon request.

\section{Acknowledgements}

We thank Kamisetty Krishna Rao for providing P. pastoris GS115 expressing $P_{\text {AOXI-GFP. This }}$ work was supported by the research grant EMR/2015/000567 and J. C. Bose Fellowship grant SB/S2/JCB-025/2015 awarded by the Science and Engineering Research Board, New Delhi, India and the research grant BT/PR30986/BRB/10/1751/2018 awarded by the Department of Biotechnology, New Delhi, India (to P.N.R). Funding from the Department of Science and Technology Fund for Improvement of S\&T Infrastructure in Higher Educational Institutions (DST-FIST), the University Grants Commission and the Department of Biotechnology (DBT)Indian Institute of Science partnership program is acknowledged. The Senior Research Fellowship to T.D. from the Department of Biotechnology, New Delhi is gratefully acknowledged.

\section{Author contributions}

T. Dey designed and performed experiments and analyzed the data. P. N. R. conceived the project, designed experiments and analyzed the data. T.D. and P.N.R. wrote the manuscript.

\section{Competing interests}

Indian Institute of Science has filed a provisional patent application for inventions related to this work. 


\section{References}

Adachi, A., M. Koizumi, and Y. Ohsumi. 2017. Autophagy induction under carbon starvation conditions is negatively regulated by carbon catabolite repression. J. Biol. Chem. 292:19905-19918. http://doi.org/10.1074/jbc.M117.817510.

Butow, R.A., and N.G. Avadhani. 2004. Mitochondrial signaling: The retrograde response. Mol. Cell. 14:1-15. http://doi.org/10.1016/S1097-2765(04)00179-0.

Cereghino, J.L., and J.M. Cregg. 2000. Heterologous protein expression in the methylotrophic yeast Pichia pastoris. FEMS Microbiol. Rev. 24:45-66. http://doi.org/10.1016/S0168-6445(99)00029-7.

Craven, R.A., D.J.F. Griffiths, K.S. Sheldrick, R.E. Randall, I.M. Hagan, and A.M. Carr. 1998. Vectors for the expression of tagged proteins in Schizosaccharomyces pombe. Gene. 221:59-68. http://doi.org/10.1016/S0378-1119(98)00434-X.

Dey, T., K.K. Rao, J. Khatun, and P.N. Rangarajan. 2018. The nuclear transcription factor Rtg1p functions as a cytosolic, post-transcriptional regulator in the methylotrophic yeast Pichia pastoris. J. Biol. Chem. 293:16647-16660. http://doi.org/10.1074/jbc.RA118.004486.

Freese, S., T. Vogts, F. Speer, B. Schäfer, V. Passoth, and U. Klinner. 2011. C-and Ncatabolic utilization of tricarboxylic acid cycle-related amino acids by Scheffersomyces stipitis and other yeasts. Yeast. 28:375-390. http://doi.org/10.1002/yea.1845

Garst, A.D., A.L. Edwards, and R.T. Batey. 2011. Riboswitches: structures and mechanisms. Cold Spring Harb. Perspect. Biol. http://doi.org/10.1101/cshperspect.a003533.

Hartner, F.S., and A. Glieder. 2006. Regulation of methanol utilisation pathway genes in yeasts. Microb. Cell Fact. 5:1-21. http://doi.org/10.1186/1475-2859-5-39. 
https://www.amazon.in/Ajinomoto-Monosodium-Glutamate-UmamiSeasoning/dp/B00IH28XDE.

https://www.fda.gov/food/food-additives-petitions/questions-and-answers-monosodiumglutamate-msg.

https://www.yeastgenome.org/locus/S000002374\#protein.

Iwama, R., and Y. Ohsumi1. 2019. Analysis of autophagy activated during changes in carbon source availability in yeast cells. J. Biol. Chem. 294:5590-5603. http://doi.org/10.1074/jbc.RA118.005698.

Jazwinski, S.M. 2014. The retrograde response: a conserved compensatory reaction to damage from within and from without. Prog. Mol. Biol. Transl. Sci. 127:133-154. https://doi.org/10.1016/B978-0-12-394625-6.00005-2

Katoh, Y., S. Nozaki, D. Hartanto, R. Miyano, and K. Nakayama. 2015. Architectures of multisubunit complexes revealed by a visible immunoprecipitation assay using fluorescent fusion proteins. J. Cell Sci. 128:2351-2362. http://doi.org/10.1242/jcs.168740.

Magasanik, B. 1993. Regulation of nitrogen utilization. COLD SPRING Harb. Monogr. Ser. 21:283. http://dx.doi.org/10.1101/0.283-317

Mara, P., G.S. Fragiadakis, F. Gkountromichos, and D. Alexandraki. 2018. The pleiotropic effects of the glutamate dehydrogenase (GDH) pathway in Saccharomyces cerevisiae. Microb. Cell Fact. 17:170. http://doi.org/10.1186/s12934-018-1018-4.

McMillan, J., Z. Lu, J.S. Rodriguez, T.-H. Ahn, and Z. Lin. 2019. YeasTSS: an integrative web database of yeast transcription start sites. Database. 2019.

Miyashita, Y., and A.G. Good. 2008. NAD(H)-dependent glutamate dehydrogenase is 
essential for the survival of Arabidopsis thaliana during dark-induced carbon starvation. J. Exp. Bot. 59:667-680. http://doi.org/10.1093/jxb/erm340.

Prielhofer, R., M. Maurer, J. Klein, J. Wenger, C. Kiziak, B. Gasser, and D. Mattanovich. 2013. Induction without methanol: novel regulated promoters enable high-level expression in Pichia pastoris. Microb. Cell Fact. 12:1-10. http://doi.org/ 10.1186/1475$2859-12-5$

Sahu, U., and P.N. Rangarajan. 2016. Methanol expression regulator 1 (Mxr1p) is essential for the utilization of amino acids as the sole source of carbon by the methylotrophic yeast, Pichia pastoris. J. Biol. Chem. 291:20588-20601. http://doi.org/10.1074/jbc.M116.740191.

Schmitt, M.E., T.A. Brown, and B.L. Trumpower. 1990. A rapid and simple method for preparation of RNA from Saccharomyces cerevisiae. Nucleic Acids Res. 18:3091-3092. http://doi.org/10.1093/nar/18.10.3091.

Shen, W., Y. Xue, Y. Liu, C. Kong, X. Wang, M. Huang, M. Cai, X. Zhou, Y. Zhang, and M. Zhou. 2016. A novel methanol-free Pichia pastoris system for recombinant protein expression. Microb. Cell Fact. 15:1-11. http://doi.org/10.1186/s12934-016-0578-4.

Silao, F.G.S., K. Ryman, T. Jiang, M. Ward, N. Hansmann, C. Molenaar, N.-N. Liu, C. Chen, and P.O. Ljungdahl. 2020. Glutamate dehydrogenase (Gdh2)-dependent alkalization is dispensable for escape from macrophages and virulence of Candida albicans. PLoS Pathog. 16:e1008328. https://doi.org/10.1371/journal.ppat.1008328

Miller, S.M., and B. Magasanik. 1990. Role of NAD-linked glutamate dehydrogenase in nitrogen metabolism in Saccharomyces cerevisiae. J. Bacteriol. 172:4927-4935. http://doi.org/10.1128/jb.172.9.4927-4935.1990 
Sutter, B.M., X. Wu, S. Laxman, and B.P. Tu. 2013. Methionine Inhibits Autophagy and Promotes Growth by Inducing the SAM-Responsive Methylation of PP2A. Cell. 154:403-415. https://doi.org/10.1016/j.cell.2013.06.041.

Vogl, T., L. Sturmberger, P.C. Fauland, P. Hyden, J.E. Fischer, C. Schmid, G.G. Thallinger, M. Geier, and A. Glieder. 2018. Methanol independent induction in Pichia pastoris by simple derepressed overexpression of single transcription factors. Biotechnol. Bioeng. 115:1037-1050. http://doi.org/10.1002/bit.26529.

Wang, J., X. Wang, L. Shi, F. Qi, P. Zhang, Y. Zhang, X. Zhou, Z. Song, and M. Cai. 2017. Methanol-Independent Protein Expression by AOX1 Promoter with trans-Acting Elements Engineering and Glucose-Glycerol-Shift Induction in Pichia pastoris. Sci. Rep. 7:1-12. http://doi.org/10.1038/srep41850.

Weber, C.A., K. Sekar, J.H. Tang, P. Warmer, U. Sauer, and K. Weis. 2020. $\beta$-Oxidation and autophagy are critical energy providers during acute glucose depletion in Saccharomyces cerevisiae. Proc. Natl. Acad. Sci. 117:12239 LP - 12248. http://doi.org/10.1073/pnas.1913370117.

Yang, J., L. Nie, B. Chen, Y. Liu, Y. Kong, H. Wang, and L. Diao. 2014. Hygromycinresistance vectors for gene expression in Pichia pastoris. Yeast. 31:115-125. http://doi.org/10.1002/yea.3001. 


\section{Figure legends}

Fig. 1 Carbon source specific, Rtg1p mediated post-transcriptional regulation of $G D H 2$ and PEPCK. (A) Epitope tagged GDH2 and PEPCK were expressed from their own promoters (schematically represented) and protein levels were examined by western blotting using anti epitope tag antibodies in the whole cell lysates of cells utilizing different carbon sources. YNBD, Yeast nitrogen base (YNB) dextrose; YNBG, YNB glycerol; YNBE, YNB ethanol; YNBA, YNB acetate and YNB Glu, YNB glutamate. (B) Expression profile of GDH2 and PEPCK mRNAs in cells utilizing different carbon compounds by qPCR. (C) Western blot analysis of epitope tagged GDH2 and PEPCK proteins synthesized from a heterologous promoter encoding $G A P D H\left(P_{G A P D H}\right)$. (D) GFP expression profile from $G D H 2\left(P_{G D H 2}\right)$ and PEPCK $\left(P_{P E P C K}\right)$ promoters in cells utilizing different carbon sources by live cell confocal imaging. (E) qPCR analysis of GFP mRNA expressed from $P_{G D H 2}$ and $P_{P E P C K}$ in cells cultured in media containing different carbon sources. (F, G) Western blot analysis of GFP synthesized from $P_{G D H 2}$ and $P_{P E P C K}$ in $G S 115$ (WT) and $\triangle r t g 1$ cells cultured in YNB Glu. The intensities of the protein bands were quantified, normalised to control (PGK) and plotted relative to GS115. PGK, phoshoglycerate kinase served as loading control. (H) qPCR analysis of GFP mRNA levels expressed from $P_{G D H 2}$ and $P_{P E P C K}$ in $\Delta r t g 1$ relative to $G S 115$ cultured in YNB Glu medium. Error bar denotes mean \pm S.D. (Biological replicates, $n=3$ ). P value is obtained from Student's t-test and is mentioned on the bar of each figure: * $P<0.05$; ** $P<0.005$; *** $P<0.0005$, ns not significant. $\mathrm{CBB}$ is the Coomassie Brillaint Blue R-stained SDSpolyacrylamide gel (A, C) serves as a loading control. Numbers (A,C,F and $\mathbf{G})$ indicate protein molecular weight markers $(\mathrm{kDa})$.

Fig. 2 Identification of Rtg1p response element within the downstream promoter region (DPR) of $\boldsymbol{P}_{G D H 2}$ and $\boldsymbol{P}_{\text {PEPCK}}$. (A) Schematic representation of DPR between the TATA box and the initiation codon of $P_{G A P D H}, P_{G D H 2}$ and $P_{P E P C K}$. (B) Western blot analysis of His tagged 
GDH2 and (C) Myc tagged PEPCK synthesied from $P_{G A P D H}$ containing $G A P D H^{D P R}$ in $G S 115$ and $\Delta r t g l$ cells cultured in YNB Glu medium. (D) Analysis of His tagged GDH2 and (E) Myc tagged PEPCK expression from chimeric $P_{G A P D H}$ containing $G D H 2^{D P R}$ and $P E P C K^{D P R}$, respectively, in GS115 and $\Delta r t g 1$ cells cultured in YNB Glu medium. Protein and mRNA levels were analysed by western blotting and qPCR respectively. The intensities of the protein bands were quantified, normalised to control (PGK) and plotted relative to GS115. Error bar denotes mean \pm S.D. (Biological replicates, $n=3$ ). $P$ value is obtained from Student's t-test and is mentioned on the bar of each figure: $* P<0.05$; ** $P<0.005$; *** $P<0.0005$, ns not significant. Numbers in (B-E) indicate molecular weight marker (kDa).

Fig. 3 Identification of oxaloacetate as the inducer of PEPCK synthesis during glutamate metabolism. (A) Protein profile of lysates of GS115, $\Delta r \operatorname{tg} 1, \Delta g d h 2$ and $\Delta p e p c k$ cultured in YNB Glu medium. Proteins were resolved on SDS-polyacrylamide gel and stained with Coomassie Brilliant Blue R. Differential expression of GDH2 and PEPCK in GS115, Artgl, $\triangle g d h 2$ and $\triangle p e p c k$ is shown. Protein bands in GS115 lane were identified as GDH2 and PEPCK by mass spectrometry in an earlier study (Dey et a., 2018). (B) Schematic representation of epitope tagged constructs of GDH2 and PEPCK expressing in GS115, $4 r t g 1, \Delta g d h 2$ and $\triangle$ pepck from $1 \mathrm{~kb}$ of $P_{G D H 2}$ and $P_{P E P C K}$, respectively. Western blot analysis of GDH2 and PEPCK levels in the whole cell protein lysates of cells cultured in YNB Glu medium using anti-His and anti-Myc antibodies, respectively. PGK served as loading control. The intensities of the protein bands were quantified, normalised to control (PGK) and plotted relative to GS115. (C) qPCR analysis of GDH2 and PEPCK mRNA levels in $\triangle p e p c k$ and $\triangle g d h 2$, respectively, relative to GS115. (D) Analysis of Myc tagged PEPCK levels in the whole cell protein lysates of GS115 and $\Delta g d h 2$ cultured in YNB Glu, YNBE and YNBA by western blotting using anti-Myc antibody. PGK served as loading control. The intensities of the protein 
bands were quantified, normalised to control (PGK) and plotted relative to GS115. (E) Schematic representation of first three reactions of glutamate utilisation pathway of $P$. pastoris catalyzed by the cytosolic enzymes GDH2, AAT2 and PEPCK. (F) Analysis of His tagged GDH2 and Myc tagged PEPCK expression in GS115 cultured in YNB Glu and YNB Akg by western blotting. The intensities of the protein bands were quantified, normalised to control (PGK) and plotted relative to GS115 cultured in YNB Glu. (G) Analysis of GFP expression from $P_{G D H 2}$ and $P_{P E P C K}$ in $G S 115$ cultured in YNB Glu and YNB Akg by live cell confocal imaging. (H) Western blot analysis of Myc tagged PEPCK levels in $\Delta g d h 2$ cultured in YNB Akg medium compared to the levels in GS115 and $\Delta g d h 2$ cultured in YNB Glu using anti-Myc antibody. PGK served as loading control. The intensities of the protein bands were quantified, normalised to control (PGK) and plotted relative to GS115 cultured in YNB Glu. (I) Analysis of expression of Myc tagged PEPCK in GS115 and Aaat2 cultured in YNB Glu and YNB Akg by western blotting using anti-Myc antibody. PGK served as loading control. The intensities of the protein bands were quantified, normalised to control (PGK) and plotted relative to GS115. (J) A model for the post-transcriptional regulation of glutamate utilisation pathway of P. pastoris. The downstream promoter region (DPR) of GDH2 and PEPCK promoters harbour putative Rtg1p response elements (RREs) between the transcription start site (TSS) and initiation codon. Upon transcription, RREs become part of the 5' UTRs of mRNAs. Translation of GDH2 mRNA requires complex interactions involving 5' UTR ${ }^{\mathrm{RRE}}$, glutamate (Glu) and Rtg1p. Translation of PEPCK mRNA requires complex interactions involving 5' UTR ${ }^{\mathrm{RRE}}$, oxaloacetate (Oaa) and Rtg1p. Akg is $\alpha$-ketoglutarate. Error bar denotes mean \pm S.D. (Biological replicates, $\mathrm{n}=3$ ). $P$ value is obtained from Student's t-test and is mentioned on the bar of each figure: $* P<0.05$; ** $P<0.005$; *** $P<0.0005$, ns not significant. Numbers in (A, B and D) and (F, H and I) indicate molecular weight marker (kDa). 
Fig. 4 GDH2 and PEPCK confer survival advantage to $P$. pastoris during carbon starvation

(A) Analysis of expression of epitope tagged GDH2 and PEPCK in GS115 subjected to carbon starvation for 6-12 h. GDH2 and PEPCK levels were examined by western blotting of cell lysates using anti-His and anti-Myc antibodies, respectively. PGK served as loading control. The intensities of the protein bands were quantified, normalised to control (PGK) and plotted relative to $0 \mathrm{~h}$ of starvation. (B) qPCR analysis of GDH2 and PEPCK mRNA levels in GS115 cultured in starvation medium. (C) Western blot analysis of GDH2 and PEPCK protein levels in cells cultured in starvation medium in the presence or absence of $1 \mathrm{mM}$ methionine. PGK served as loading control. The intensities of the protein bands were quantified, normalised to control (PGK) and plotted relative to $0 \mathrm{~h}$ of starvation. (D) Analysis of viability GS1 15, $\Delta g d h 2$ and $\Delta p e p c k$ upon carbon starvation by cell survival assay. At indicated time points, nutrientdeprived cultures were removed and plated on YPD agar containing yeast extract (1\%), peptone (2\%) and dextrose (1\%) . (E) Quantitation of cell survival (D). Number of colonies on the agar plates were counted and percent colony forming unit was plotted as percent survival. Error bar denotes mean \pm S.D. (Biological replicates, $\mathrm{n}=3$ ). $P$ value is obtained from Student's t-test and is mentioned on the bar of each figure: $* P<0.05$; ** $P<0.005$; *** $P<0.0005$, ns not significant. Numbers in $\mathbf{A}$ and $\mathbf{C}$ indicate molecular weight marker (kDa).

Fig. 5 A monosodium glutamate (MSG)-inducible Pichia pastoris expression system. (A) GFP expression profile from $P_{G D H 2}$ and $P_{P E P C K}$ in cells cultured in media containing either glycerol, glutamate or MSG as the sole source of carbon by live cell confocal imaging. (B) Comparative analysis of GFP expression from $P_{A O X 1}, P_{G D H 2}$ and $P_{P E P C K}$ in cells cultured in YNBM $\left(P_{A O X I}\right)$ and YNB MSG $\left(P_{G D H 2}\right.$ and $\left.P_{P E P C K}\right)$ by western blotting. $(\mathbf{C})$ GST tagged antiGFP nanobody mediated pull down assay. GST-tagged anti-GFP nanobodies obtained from $E$. 
coli lysates (Katoh et al., 2016) were incubated with glutathione-sepharose beads and $P$. pastoris cell lysates as indicated. After washing, proteins bound to glutathione-sepharose beads were resolved on SDS-PAGE and visualized by Coomassie Brilliant Blue staining. (D) Quantitation of GFP levels in (B) (left panel) and (C) (right panel). The intensities of the protein bands were quantified and normalised to GFP expressed from $P_{A O X I}$ and YNBM cultured cells. Error bar denotes mean \pm S.D. (Biological replicates, $\mathrm{n}=3$ ). $P$ value is obtained from Student's t-test and is mentioned on the bar of each figure: * $P<0.05$; ** $P<0.005$; *** $P<0.0005$, ns not significant. (E) Estimation of amount of GFP in (C) using BSA standard curve generated from known concentrations of BSA. (F) Pictorial representation of a glutamate inducible expression vector containing two glutamate inducible expression cassettes. Gene of interest (GoI) can be expressed from $G D H 2$ and $P E P C K$ promoters simultaneously in the same $P$. pastoris strain maximising recombinant protein yield. Numbers in $(\mathbf{B})$ and $(\mathbf{C})$ indicate protein molecular weight markers (kDa). Ponceau S-stained blot (B) serves as loading control. 
A

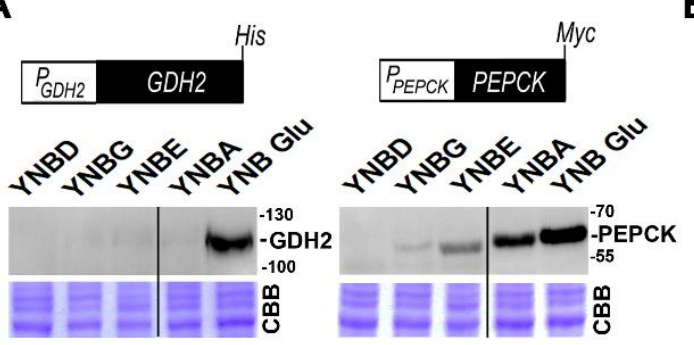

C

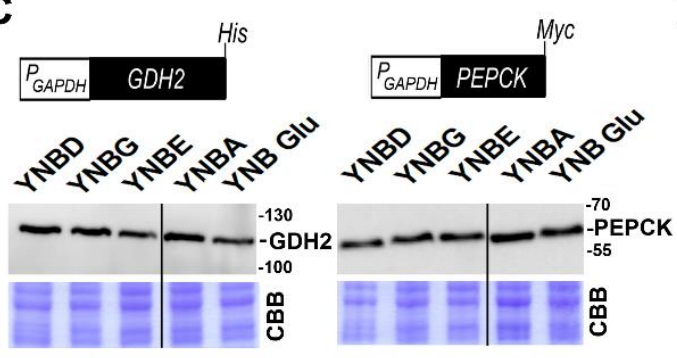

D

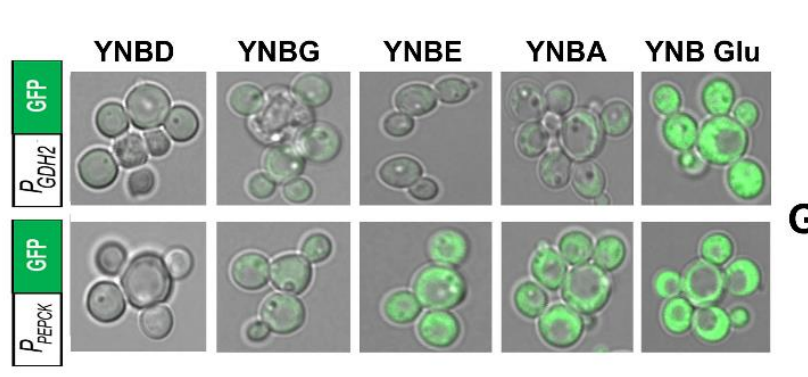

B

E
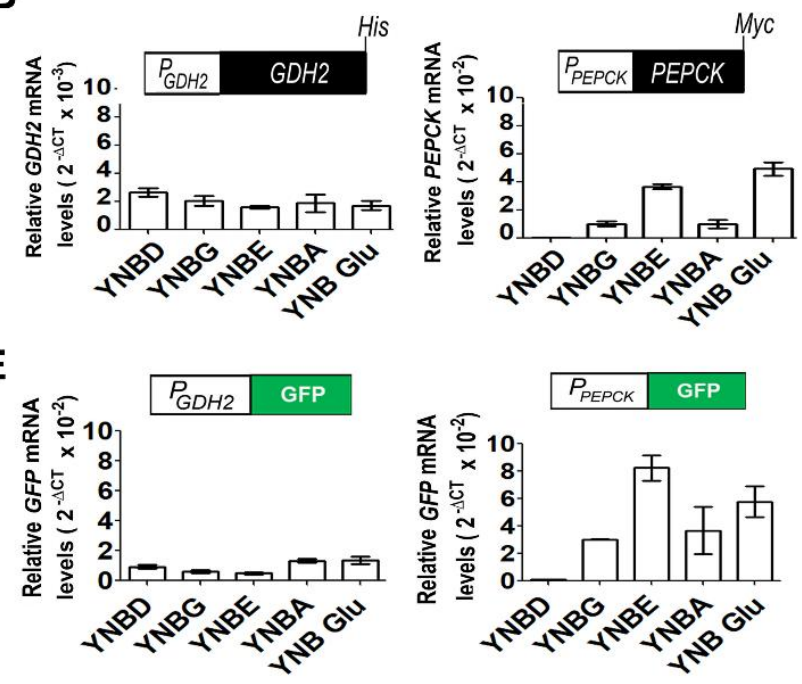

$\mathbf{F}$
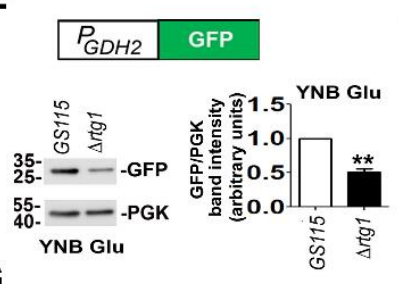

H
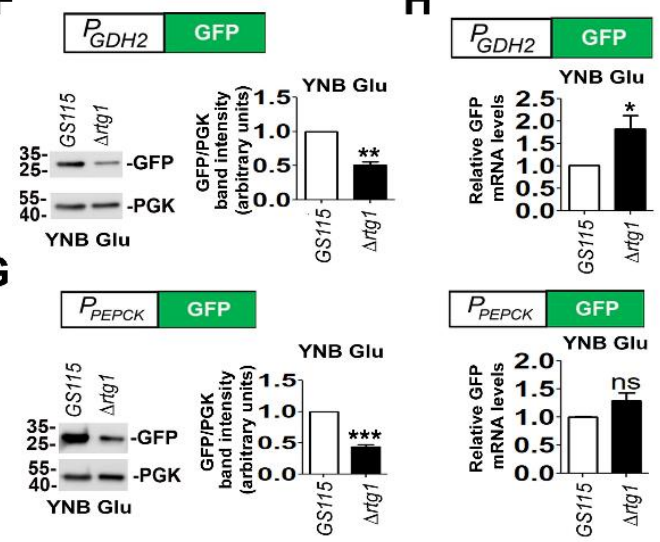

Fig.1 
A

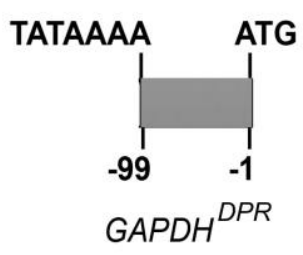

B

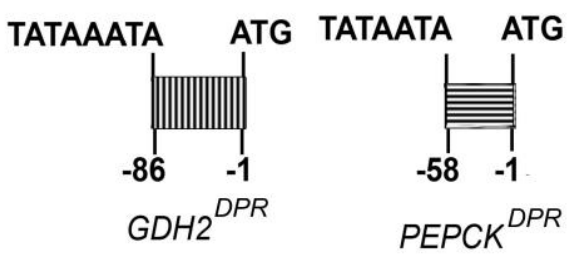

C
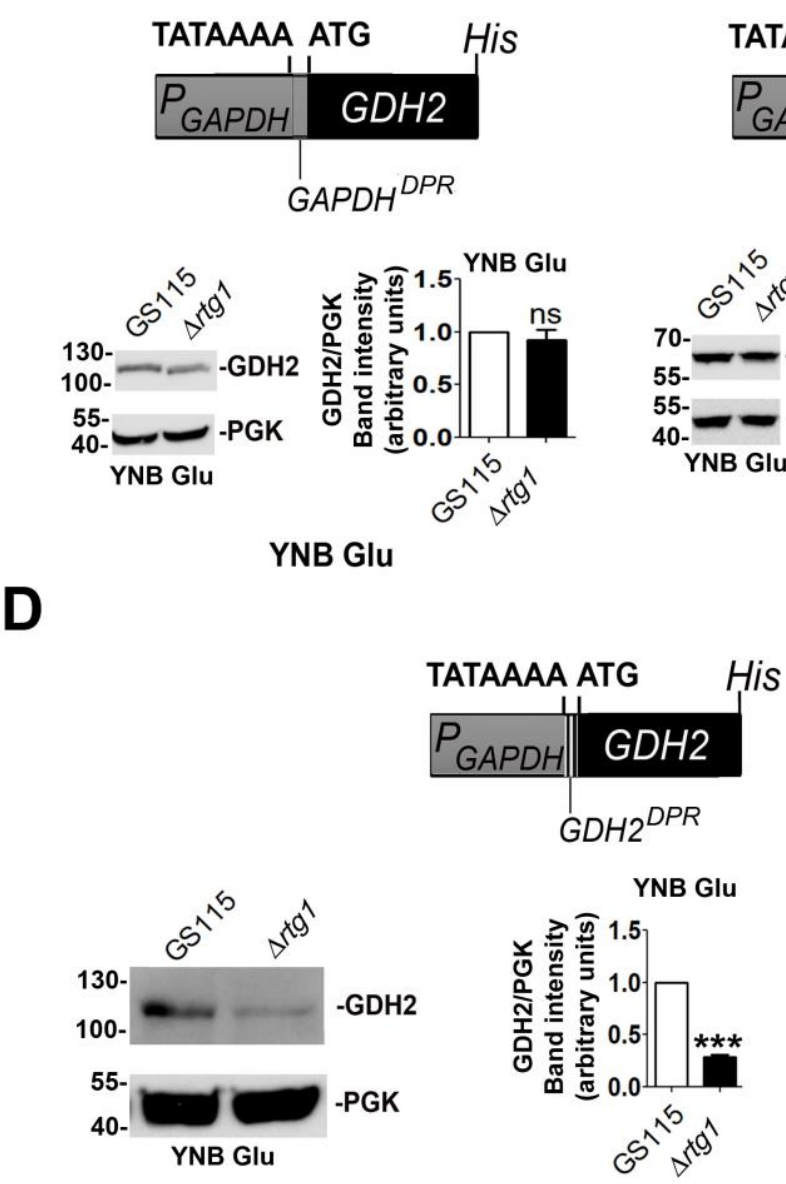

E
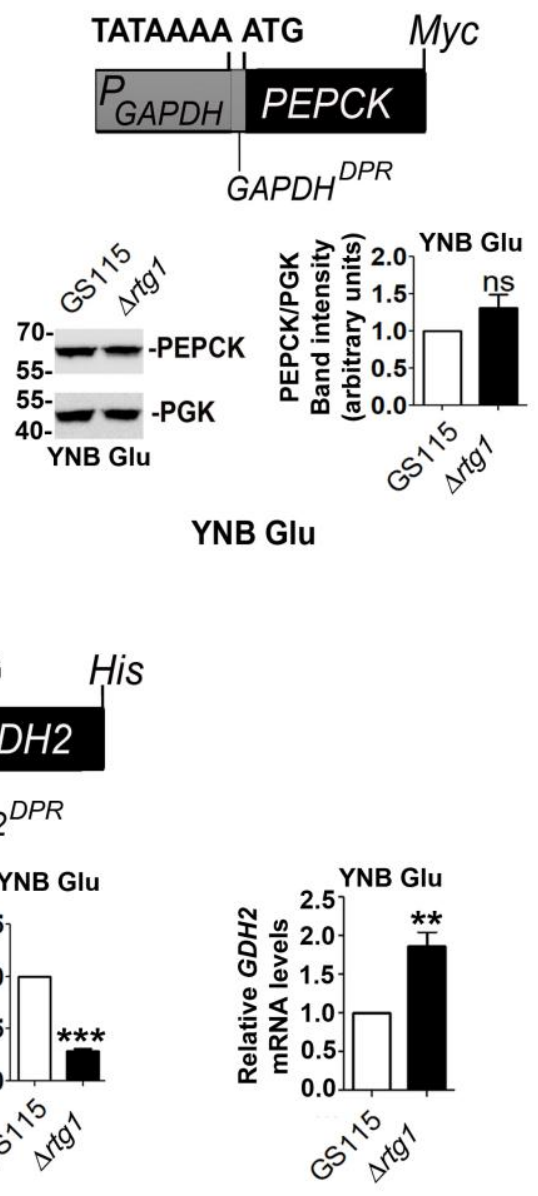
A

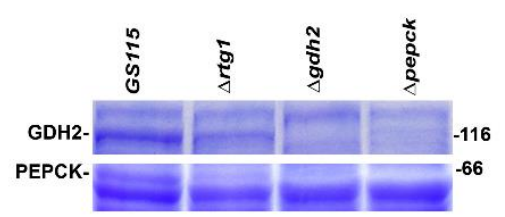

B

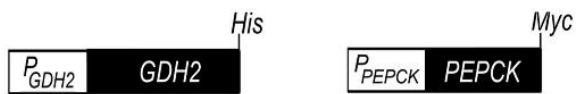

C
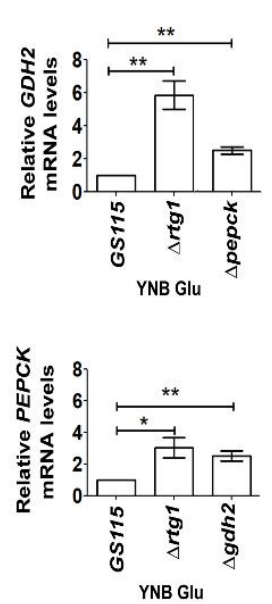

$\mathbf{E}$

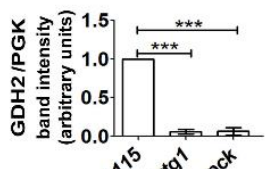

$\mathbf{F}$

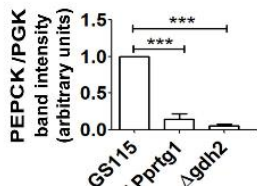

G
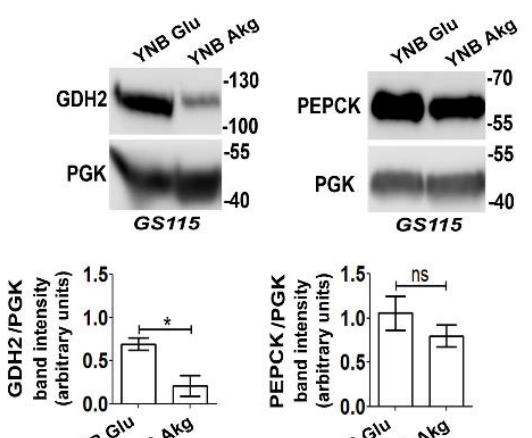

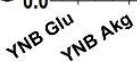

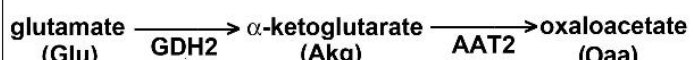

$\downarrow$ PEPCK

Phosphoenolpyruvate

H
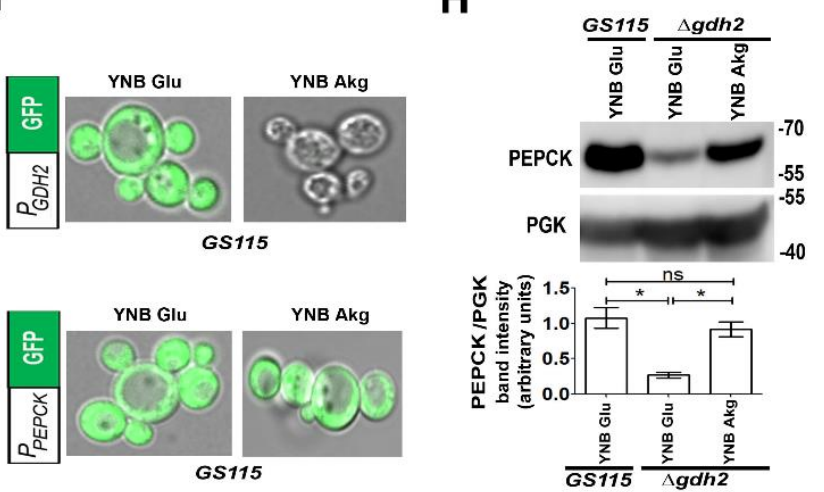

I

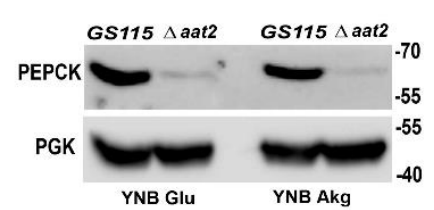

J

TATAAAA|ATG

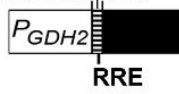

$\frac{\text { GS115 } \Delta a a t 2}{\text { YNB Glu }} \frac{\text { GS115 } \Delta a a t 2}{\text { YNB Akg }}$
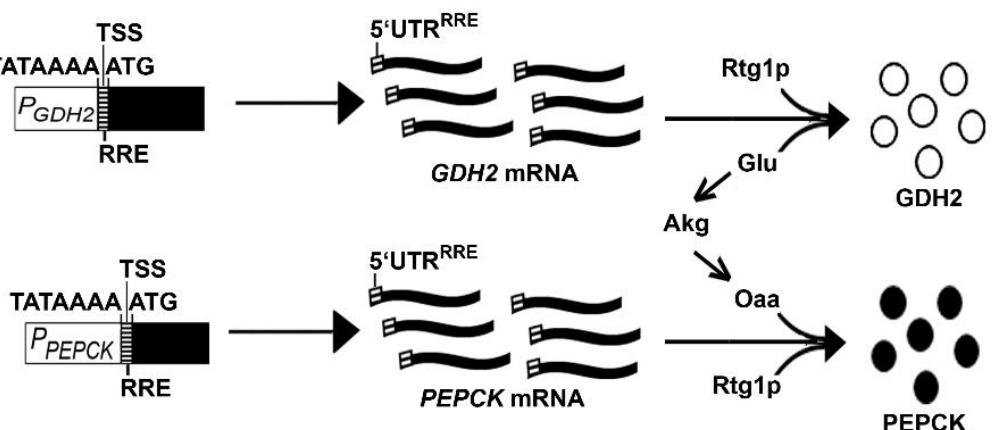

Fig. 3 
A
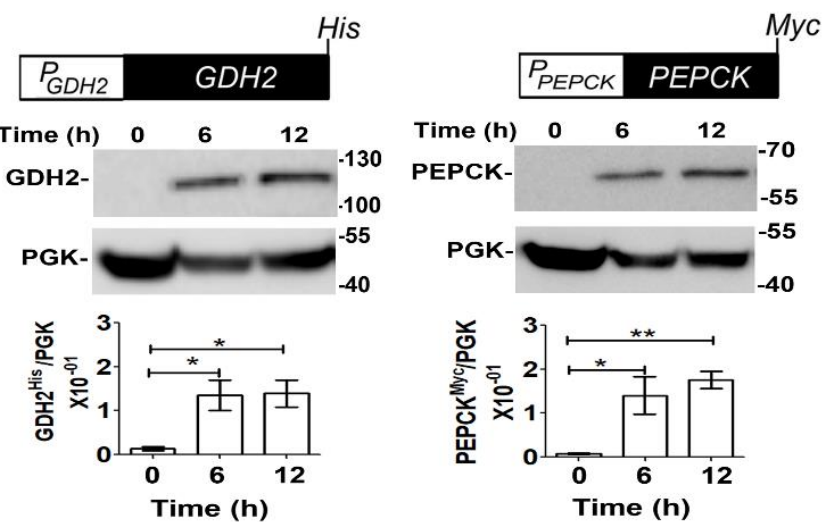

B
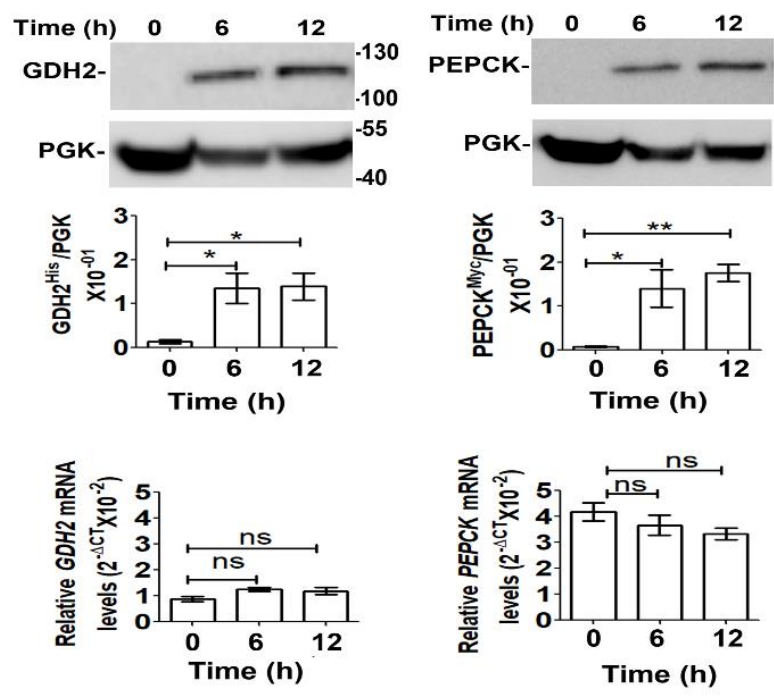

C
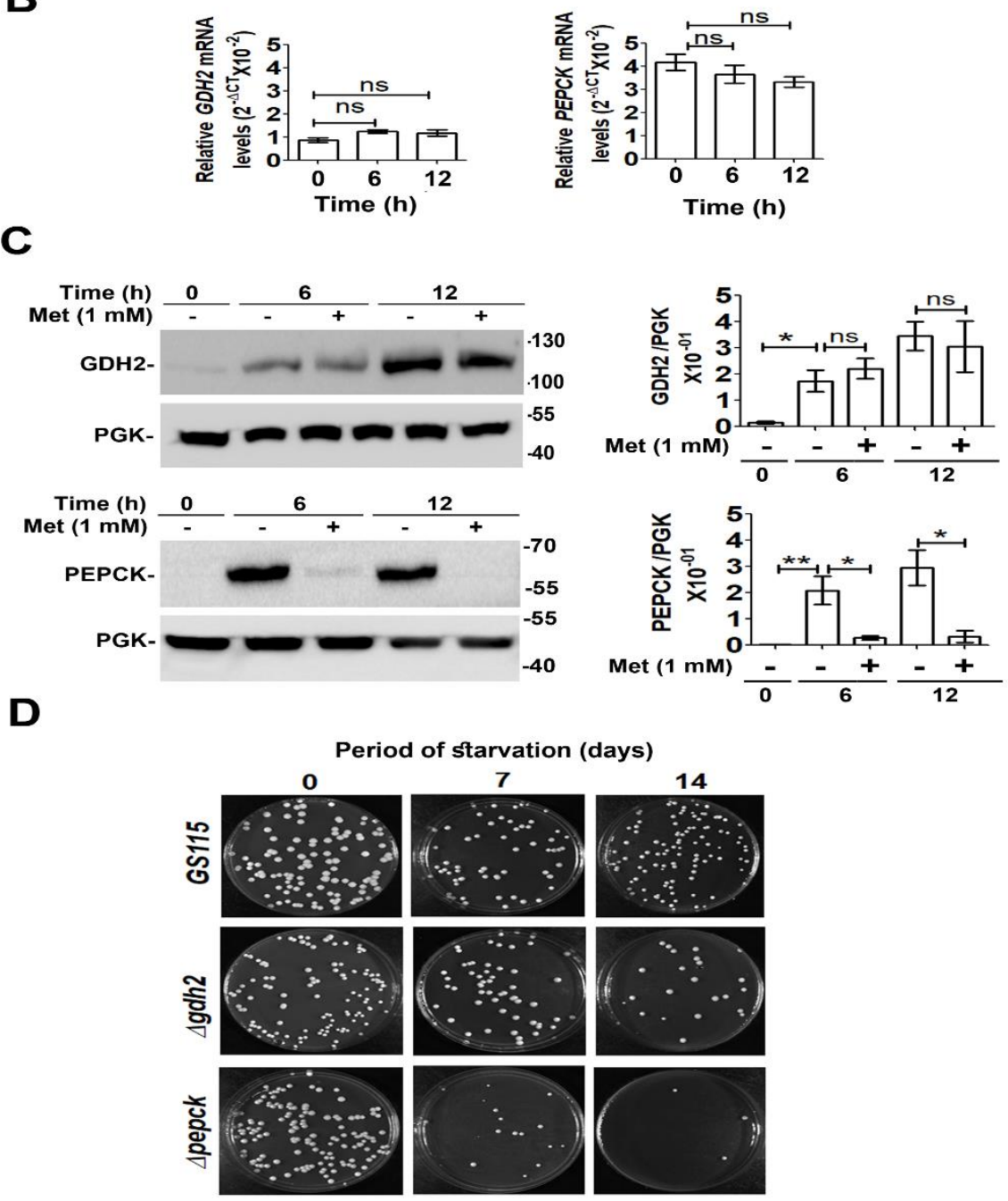

E

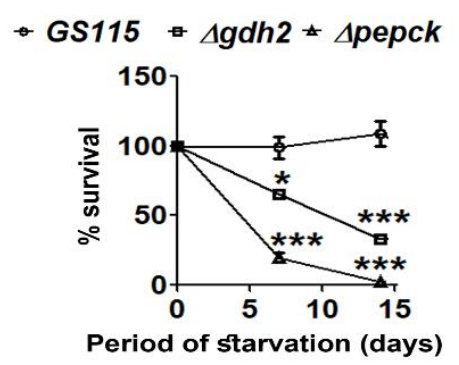

Fig. 4 
A
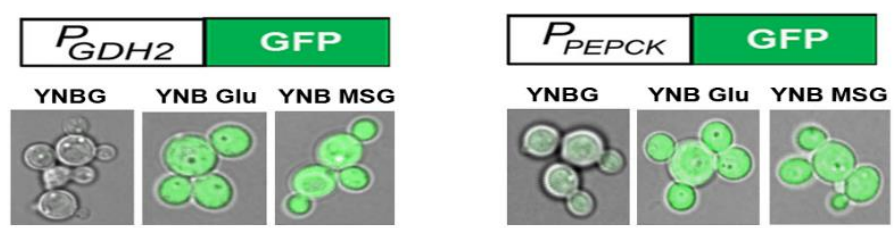

B

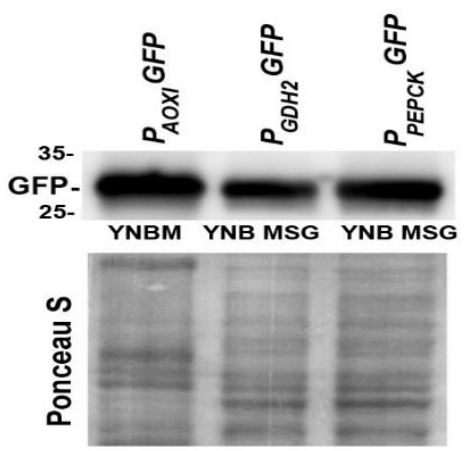

C

D
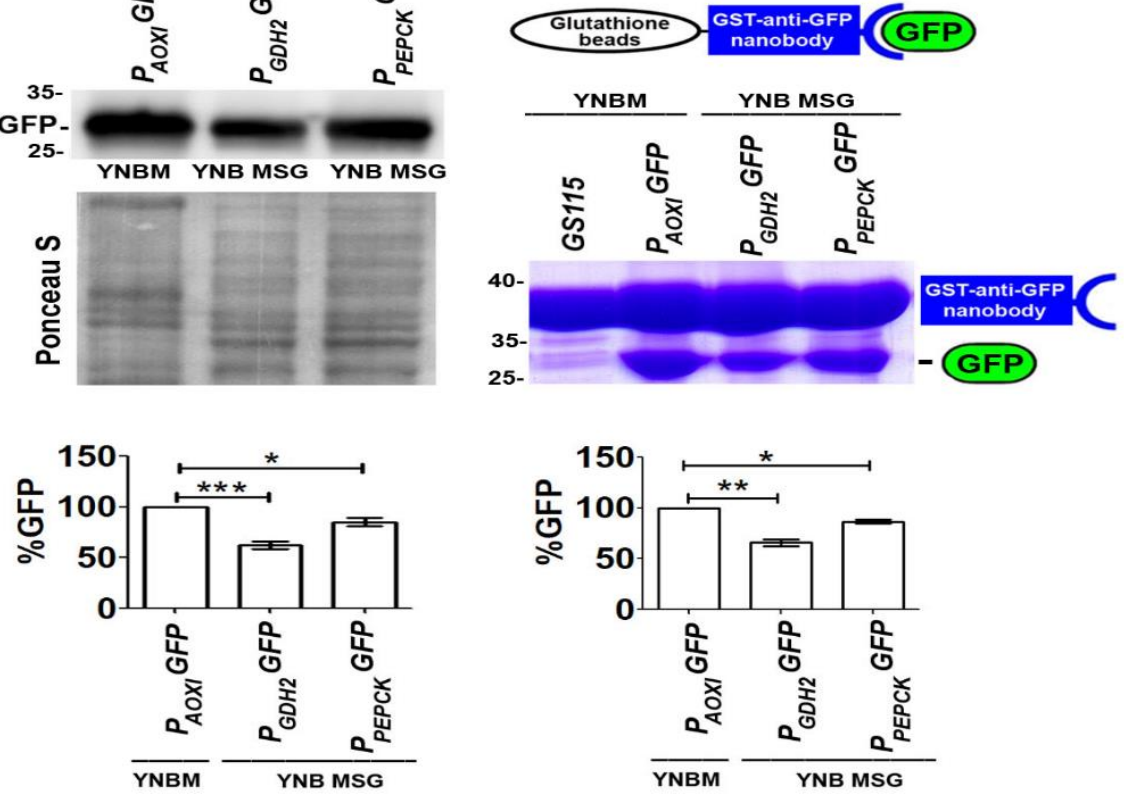

$\mathbf{E}$
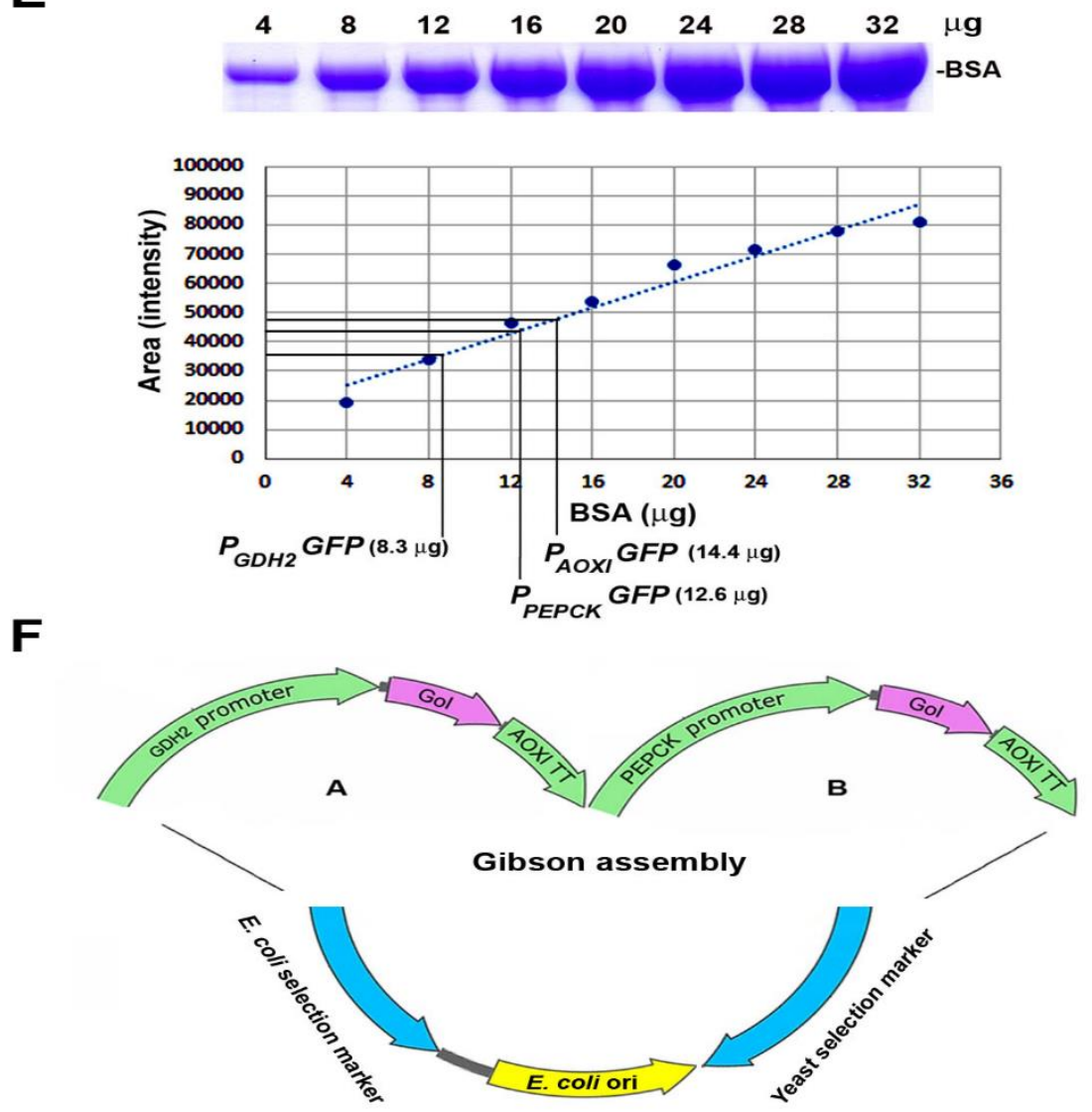

$\mathbf{F}$

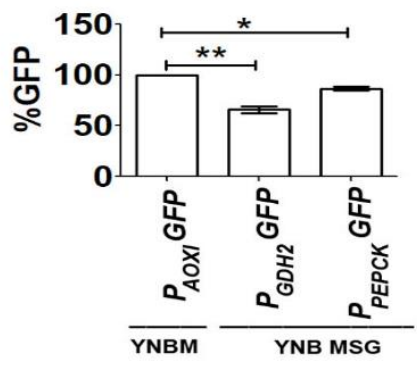

Fig.5 